\title{
The acute abdomen in spinal cord injury individuals
}

\author{
Z Bar-On and A Ohry \\ Neurological Rehabilitation Department, Sheba Medical Center, Tel Hashomer 52621, Affiliated to the Sackler \\ School of Medicine, Tel Aviv University, Israel.
}

A review of 1300 patients with spinal cord injury (SCI), over a period of 14 years, revealed 12 patients with an 'acute abdomen'. Seven events occurred during the initial admission, ranging from 10 days to 9 months from injury, and five during readmission of 'chronic' SCI patients. Four were in the acute stage 10-30 days from injury, all with peptic ulcer perforations. The remainder had either an intestinal obstruction, appendicitis or peritonitis. All of the neurological levels were above T6 except for one patient who had a low level paraplegia. The classical signs of an 'acute abdomen' may be missing in such patients thus delaying diagnosis by 1-4 days. The most important signs were autonomic dysreflexia, referred shoulder tip pain, abdominal pain, abdominal distension, increased spasticity and abdominal pain with nausea and vomiting. Less importance was given to the classical signs of abdominal tenderness, abdominal muscle rigidity, rebound, fever and of leukocytosis. Prompt diagnosis and treatment will minimise morbidity and mortality.

Keywords: spinal cord injury; acute abdomen; volvulus; perforation; autonomic dysreflexia

\section{Introduction}

Diagnosing an 'acute abdomen' is one of the critical problems facing surgeons and can be particularly difficult in a SCI patient.

A silent atypical acute abdomen, ${ }^{1}$ and a delayed diagnosis ${ }^{2}$ have been reported in the geriatric population. The diagnosis is even more complex in SCI patients who are also known to present with peculiar septic responses. ${ }^{3}$

SCI patients with neurological levels above T6 have altered physiological responses and loss of sensory, motor and reflex functions. The response to visceral pathology depends on the level and degree of the cord lesion, whether it is complete or incomplete and whether the reflex arc below the cord lesion is intact. ${ }^{4,5}$ The typical signs of an acute abdomen, such as abdominal muscle rigidity, tenderness and peritoneal rebound are usually absent in those with a high cord lesion. Pyrexia and leukocytosis may also be absent. ${ }^{3,6}$ The presentation of an 'acute abdomen' in SCI patients may thus puzzle attending physicians and surgeons. In the acute stage of spinal shock, we must rely on laboratory and radiological data as well as on the vital signs. ${ }^{7}$ Following the return of spinal reflexes, patients with high cord lesions above T6 with an acute abdomen may present with autonomic dysreflexia (AD). ${ }^{8}$ In these patients autonomic dysreflexia is usually due to distension of a hollow viscus such as the urinary bladder, stomach, bowel or uterus or due to perforation of a viscus. $\mathrm{AD}$ is a life-threatening emergency in its own right. ${ }^{9}$
Another sign that is manifested by SCI patients with an acute abdomen is referred shoulder tip pain, mainly in those with a viscus perforation, ${ }^{7,10-12}$ but can also appear in patients with basal pneumonia. Abdominal pain, if present, is usually dull, oppressive and poorly localised. Although some SCI patients will have better visceral sensation than others, this dull visceral pain is mediated either by the vagus ${ }^{13}$ or by the sympathetic paravertebral chain entering the cord higher than the lesion, ${ }^{14}$ or with the bulk of the information passing through the spinothalamic tracts. ${ }^{10}$ This abdominal pain is not usually suggestive of an abdominal emergency unless it is preceded or is followed by anorexia, nausea and vomiting. Increased spasticity should also raise suspicion of abdominal disease especially if it is associated with abdominal pain. ${ }^{4,5,7,10}$ Patients with a low thoracic injury experience pain similar to that noted by able bodied people, but their increased tendency to complications such as urinary tract infections and pressure sores, may delay the prompt diagnosis of an acute abdomen.

Laboratory findings are not reliable, because most patients with SCI have leukocyturia at some stage, and many do not react with leukocytosis, thus attributing non-specific symptoms to the genitourinary tract.

Radiographic studies, including chest films, abdominal series, oral cholecystograms, CT scans, sonograms and barium studies were shown to lead to the correct diagnosis in $77 \%$ of the patients presented by Neumayer. ${ }^{14}$

The problem of a delayed diagnosis, causing morbidity and mortality has been well documented, with a mortality rate of $10-15 \%$. 


\section{Material and methods}

During the 14 year period of 1978-1991, 12 SCI patients out of about 1300 admissions developed an 'acute abdomen'. Seven of them developed this during the initial admission, ranging from 10 days to 9 months following the injury; of these four developed it within 10-30 days coinciding with the period of spinal shock. Five patients were readmitted with an acute abdomen 2-19 years following the injury. In all but one of these patients the neurological level was above T6. The only patient with a level below T6 had become paraplegic following surgery for a dissecting aortic aneurysm, and developed massive haemorrhage from erosive gastritis, 30 days following the onset of paraplegia, and 1 day following overseas transfer to our unit. He presented with melena. The abdominal pathology can be divided into four groups. The acute stage group (10-30 days) all had perforated ulcers (two duodenal ulcers and two gastric ulcers) and one had a fatal outcome from massive bleeding, leading to brain death. The delay in diagnosis in this group was 1-4 days. The second distinct group consisted of four patients with intestinal obstruction: one large bowel; one volvulus of the bowel; and two with volvulus of the small bowel with necrosis, in one fatal due to massive necrosis. These patients were 9 months to 19 years post injury. Intestinal obstruction may be due to long standing constipation and altered colonic motility, which is well known in SCI individuals. ${ }^{15}$ The third group consisted of three patients who were diagnosed at operation as having acute appendicitis, with 1-3 days delay in diagnosis. The fourth group consisted of one patient who had peritonitis with free air seen on an abdominal radiograph and referred shoulder tip pain; but only massive air release on opening the peritoneum without detecting the exact origin of the perforation. Ten patients recovered well from surgery; however two of these patients died a few months later, one from chest complications and sepsis, and the other patient $(\mathrm{C} 2$ complete) from respiratory failure.

Two patients died from their abdominal disease, one because of misdiagnosis of a volvulus with massive bowel necrosis, the other from brain death due to massive bleeding ( $12 \mathrm{~h}$ and 10 days postoperatively, respectively).

Most of our patients had a plain abdominal radiograph, which was normal in three patients: two patients had evidence of free air in the peritonial cavity, and dilatation of bowel loops in six patients.

Only three patients had leukocytosis. Eight had a fever, late in the disease process, about 1 day prior to diagnosis. One patient had complained of suffocation, whilst on full mechanical ventilation, and developed acute abdominal distension from a gastric perforation. In most of our patients the diagnosis of an acute abdomen was made on clinical grounds, and as Com$\operatorname{arr}{ }^{16}$ suggested 'when in doubt, take it out'. We felt that an early laparotomy was safer than was hesitancy.

Our last patient died in June 1991 from massive bowel necrosis and volvulus symptoms for only $24 \mathrm{~h}$. $\mathrm{He}$ had presented with autonomic dysreflexia followed by vomiting, referred shoulder tip pain, increased spasticity, fever and haemodynamic deterioration, suspicious of a perforation. It was felt that subsequent delay in surgery contributed to the death of this patient.

\section{Discussion}

The problem of the acute abdomen in SCI individuals has been recognised since the Second World War. Abdominal emergencies are quite rare in such patients, but prompt diagnosis minimises morbidity and mortality.

Hoen in $1948^{17}$ and Greenfield in $1949^{18}$ each described six cases. Greenfield based the diagnosis on routine clinical and laboratory surveys, and suggested that patients with chronic paraplegia withstood abdominal operations well. Hoen had six patients with cord lesions $\mathrm{C} 6$ to $\mathrm{T} 8$ with problems of renal or bladder origin, gastric ulcer responding to medical management, intestinal adhesions and fecal impaction.

O'Hare $^{16}$ in 1966 reported 15 'pure abdominal patients' in 13 years, and concluded that in those with a suspected obstruction early laparotomy was safe. The largest groups were reported by Juler and Eltorai ${ }^{7}$ in 1985 , and by Neumayer ${ }^{13}$ in 1990 . Juler reported 37 patients with neurological levels of C3-L5 (32 complete) 10 with acute appendicitis, 11 with a perforated viscus and 16 with a bowel obstruction. They emphasised the importance of the features of autonomic dysreflexia and of shoulder tip pain. Neumayer, however, in his 22 patients based the correct diagnosis in $77 \%$ on the radiographic findings.

\section{Conclusion}

Early recognition of the clinical manifestations of acute abdominal emergencies in SCI patients, especially autonomic dysreflexia, referred shoulder tip pain, increased spasticity and abdominal pain with nausea and vomiting and abdominal distension may lead to a prompt diagnosis.

We suggest that the evaluation of tetraplegic and paraplegic patients by a spinal injury specialist is essential for the early diagnosis and treatment of an acute abdomen and for the reduction of morbidity and mortality in these patients.

\section{References}

1 Block MA. Managing the silent, atypical acute abdomen. Geriatrics 1983; 38: 50-60.

2 Fulton JD, Peebles SE, Smith GD, Davie JW. Unrecognized viscus perforation in the elderly. Age Ageing 1989; 18: 403-406.

3 Ohry A, Heim M, Rozin R. Peculiar septic responses in traumatic tetraplegic patients. Paraplegia 1983; 21: 318-321.

4 Charney KJ, Juler GL, Comarr AE. General surgery problems in patients with spinal cord injuries. Arch Surg 1975; 110: 1083-1088.

5 Juler GL. Acute abdominal emergencies in spinal cord injury patients. J Am Paraplegia Soc 1979; 2: 1-5.

6 Sugarman B, Brown D, Musher D. Fever and infection in spinal cord injury patients. JAMA $1982 ; 248$ : 66-70.

7 Juler GL, Eltorai IM. The acute abdomen in spinal cord injury 
patients. Paraplegia 1985; 23: 118-123.

8 Guttmann L, Whitteridge D. Effects of bladder distension on autonomic mechanisms after spinal cord injuries. Brain 1947; 70: $361-405$.

9 Eltorai I, Kim R, Vulpe M, Kasravi H, Ho W. Fatal cerebral hemorrhage due to autonomic dysreflexia in a tetraplegic patient: case report and review. Paraplegia 1992; 30: 355-360.

10 Miller LS, Staas WE, Herbison GJ. Abdominal problems in patients with spinal cord lesions. Arch Phys Med Rehabil 1975; 56: 405-408.

11 Kewalramani LS. Neurogenic gastroduodenal ulceration and bleeding associated with spinal cord injuries. J Trauma 1979; 19: $259-265$.

12 Walsh JJ, Nuseibeh I, El-Masri W. Perforated peptic ulcer in paraplegia. Paraplegia 1974; 11: 310-313.
13 Crawford JP, Frankel HL. Abdominal 'visceral' sensation in human tetraplegia. Paraplegia 1971; 9: 153-158.

14 Neumayer LA, Bull DA, Mohr JD, Putnam CW. The acutely affected abdomen in paraplegic spinal cord injury patients. Ann Surg 1990; 212: 561-566.

15 Aaronson MJ, Freed MM, Burakoff R. Colonic myoelectric activity in persons with spinal cord injury. Dig Dis Sci 1985; 30 295-300.

16 O'Hare JM. The acute abdomen in spinal cord injury patients. Proc Ann Clin Spinal Cord Inj Conf 1966; 15: 113-117.

17 Hoen TI, Cooper IS. Acute abdominal emergencies in patients with spinal cord lesions. Arch Phys Med Rehabil 1948; 49 343-348.

18 Greenfield J. Abdominal operations on patients with chronic paraplegia. Arch Surg 1949; 59: 1077-1087. 